\title{
The lung cancer research career of Prof. Ramón Rami-Porta: passion, vision and reflection
}

Submitted Sep 20, 2017. Accepted for publication Sep 25, 2017.

doi: $10.21037 /$ jtd.2017.09.137

View this article at: http://dx.doi.org/10.21037/jtd.2017.09.137

As co-coordinator of the Bronchogenic Carcinoma Cooperative Group of the Spanish Society of Pneumology and Thoracic Surgery and Chair of the Staging and Prognostic Factors Committee of the IASLC, Prof. Ramón Rami-Porta has been dedicated to lung cancer staging research for almost 35 years. Well-known of his contribution in fighting with lung cancer, Prof. Rami-Porta is very dynamic both in the clinical practice and academic arena.

'Choose a job you love and you will never have to work a day in your life'. Looking back, Prof. Rami-Porta is fortunate to say that he has never regretted to have chosen thoracic surgery. It is a hard specialty, but Prof. Rami-Porta perceives himself in permanent vacation, because he really loves it, with its lights and its shadows.

Prof. Rami-Porta told us, to become a successful surgeon, one has to know its profession well. This means to study hard, to practice regularly and to constantly update one's knowledge. The surgeon, as a specialist, is supposed to know a lot about his particular field, but must not forget to see the patient as a unique person with individual values and needs. The surgeon must do so with honesty, modesty and compassion.

To know more, please refer to the following in-depth interview with Prof. Rami-Porta by AME (Figure 1).

\section{Introduction of Dr. Ramón Rami-Porta (Figure 2)}

Ramón Rami-Porta graduated from Barcelona University Medical School in 1980. He followed specialty training in thoracic surgery at the Jiménez Diaz Foundation, Madrid, and was board-certified in thoracic surgery in 1985 . During his residency he also worked on a research project on malignant pleural effusion for his $\mathrm{PhD}$ degree that he received in 1986 from the Autonomous University of Madrid. In the last years of his medical training and during his residency, he visited several institutions in the United States of America for further training, and, as a certified thoracic surgeon, he was an overseas trainee at the National

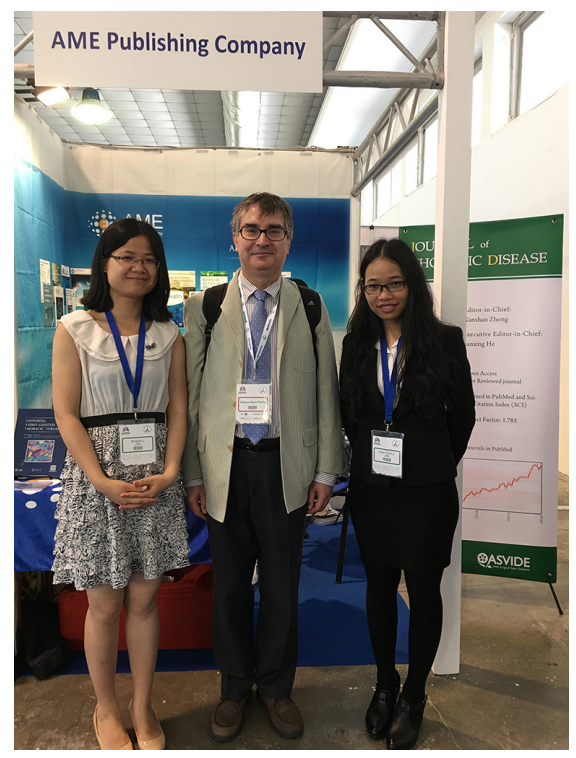

Figure 1 AME editors met with Prof. Rami-Porta in the ESTS 2016 annual meeting.

Cancer Centre in Tokyo. As a thoracic surgeon he worked in Barcelona and in Santiago de Compostela. Since 1989, he has been working at Hospital Universitari Mútua Terrassa, in Terrassa, where he is Clinical Chief of the Thoracic Surgery Service. His major interests are lung cancer staging, surgical treatment of lung cancer, and thoracic oncology in general. He is a member of several national and international medical societies. In the Spanish Society of Pneumology and Thoracic Surgery, he was a member of the group of coordinators of the Bronchogenic Carcinoma Cooperative Group (GCCB-S), a multicentre group whose project was to study lung cancer staging. He has been member of the International Association for the Study of Lung Cancer since 1990, is a member of its Staging and Prognostic Factors Committee, was Chair of this committee from 2009 to 2016, and is, at present, its Past-Chair. He also is a member of the Network of Centers for Biomedical Research in Respiratory Diseases (CIBERES) Lung Cancer 


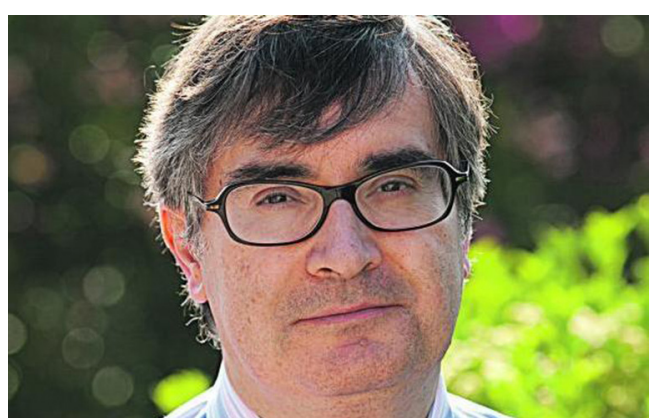

Figure 2 Prof. Ramón Rami-Porta.

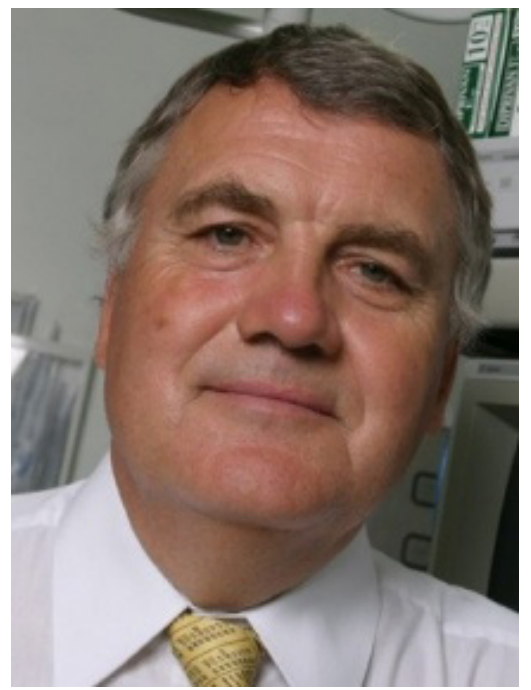

Figure 3 Prof. Peter Goldstraw.

Group, a government-supported network study group on lung cancer.

\section{Get a close look at lung cancer staging}

AME: It is well-known that you have made significant and dedicated contributions in lung cancer staging. Looking back, how did you get started with lung cancer staging research?

Dr. Rami-Porta: My interest in lung cancer staging can be traced back to my residency years, in the early 1980s. My chief, then, asked me to prepare a lecture on lung cancer staging for the medical students who were rotating in our service. I think all started there, some 35 years ago. A few years later, I became a member of the Bronchogenic Carcinoma Cooperative Group of the Spanish Society of Pneumology and Thoracic Surgery and was appointed coordinator of the Group together with a pulmonologist, Dr. Ángel López Encuentra, and a thoracic surgeon, Dr. José Luis Duque Medina. We all had a very special interest in lung cancer. The Group had the objective to study and revise the tumor, node and metastasis (TNM) classification of lung cancer based on Spanish data. In four years, nearly 3,000 patients who had undergone lung resection for lung cancer were registered prospectively. At about the same time, I became a member of the International Association for the Study of Lung Cancer (IASLC). After the constitution of the IASLC Staging and Prognostic Factors Committee (SPFC), Prof. Peter Goldstraw (Figure 3), the first Chair of this Committee, invited me to join in. At due time, the database of the Bronchogenic Carcinoma Cooperative Group was transferred to the newly created IASLC International Database. I later became Vice-Chair and then Chair of the SPFC at the end of Prof. Goldstraw's term (Figure 4).

AME: As the Chair of the Staging and Prognostic Factors Committee of the IASLC, could you share with us how important staging is and what is its role in lung cancer treatment (e.g., surgery)?

Prof. Rami-Porta: It is important to keep in mind that the TNM classification and staging system determine the anatomic extent of the tumor, that is, its size, its location, the structures it invades, and whether there is nodal involvement or metastatic spread. The TNM classification is pure anatomy, but by describing the anatomy and analyzing survival we can assess the prognosis of the disease before and after treatment. Clinical guidelines on therapy, derived from clinical trials, are based on anatomic stage. The staging system is not only useful to surgeons to indicate tumor resection; it is also useful to radiotherapists, because they need to know the extent of the disease; and it is important to medical oncologists to indicate chemotherapy and targeted therapies. It is of paramount importance to those patients who will receive multimodality therapy. All specialists involved in managing lung cancer patients benefit from this staging system.

AME: Now we are looking at the 8th edition lung cancer staging system, which has experienced 7 significant updates. How did you see the evolvement of lung cancer staging system?

Prof. Rami-Porta: So far, in my professional life, I have 


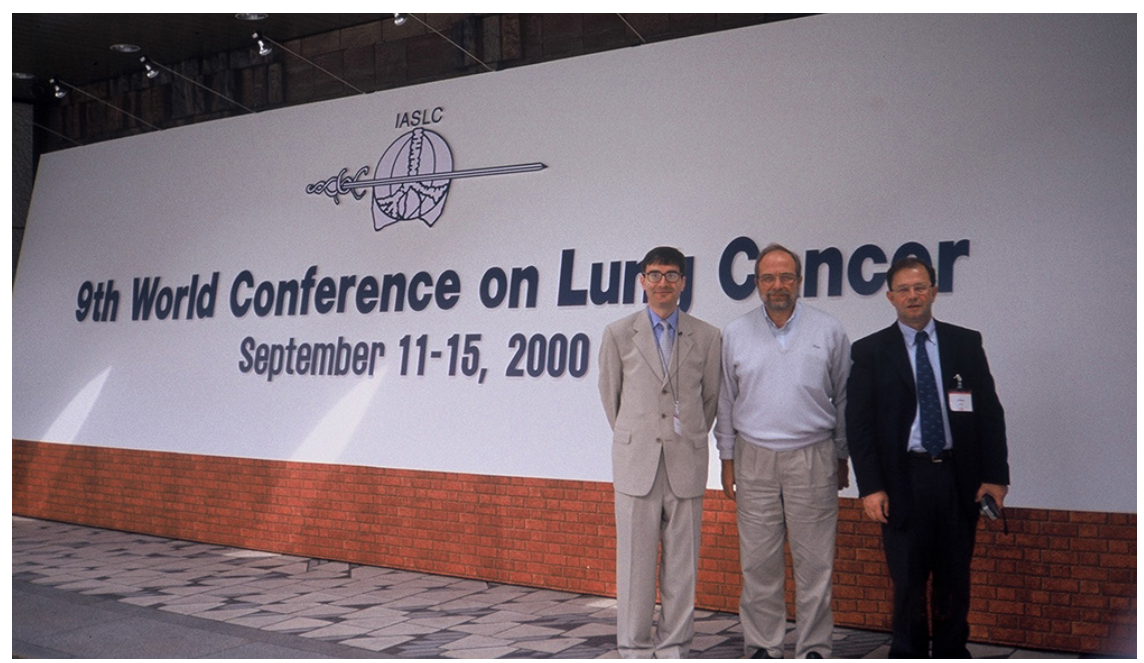

Figure 4 Showing from left to right, Drs. Ramrta in the ESTSÁngel Lgpez Encuentra and José Luis Duque Medina, was taken at the venue of the World Conference on Lung Cancer, Tokyo, Japan, 2000. The three of us were the Coordinators of the Bronchogenic Carcinoma Cooperative Group of the Spanish Society of Pneumology and Thoracic Surgery.

used six editions of the TNM classification for lung cancer, but the first edition was published by the Union for International Cancer Control (UICC) in 1968. Prof. Peter Goldstraw, who edited the first edition of the IASLC Staging Manual in Thoracic Oncology [2009], did a lot of research on the history of the classification and wrote a chapter for the Manual that is fundamental to understand the development of the classification. Because of its quality and relevance, this historical chapter has been kept unaltered in the second edition of the IASLC Staging Manual in Thoracic Oncology [2016]. Its reading is essential to all those interested in the topic. In brief, the first editions assigned anatomic descriptors to categories mainly intuitively. The era of data-based revisions started with Prof. Clifton Mountain, who over the years built a lung cancer database that he used to revise the classification. The $4^{\text {th }}, 5^{\text {th }}$ and $6^{\text {th }}$ editions were informed by this database. However, this database had its limitations: it was exclusively North American and all patients had undergone surgical treatment. In 1996, shortly before the publication of the $6^{\text {th }}$ edition, Prof. Goldstraw proposed the collection of a large, international database within the IASLC to revise future editions of the classification. So far, two databases of nearly 100,000 patients each have been used to inform the $7^{\text {th }}$ and the $8^{\text {th }}$ editions of the classification. The IASLC now provides the UICC and the American Joint Committee on Cancer (AJCC) with evidences to recommend changes in the revisions. It goes without saying that the recommendations of the IASLC are very robust, because they are based on a large number of patients treated with all sorts of treatment modalities in many countries around the world, and on sound methodology and statistical analyses. The move to data-driven revisions has been essential because the proposed recommendations for changes are solidly based.

Anatomic extent is a good prognosticator, but it does not explain all prognosis. The plan for the $9^{\text {th }}$ edition, due to be published in 2024, is to further revise the anatomic descriptors, but also to combine anatomic and nonanatomic parameters, such as blood analyses, co-morbidity, and molecular markers, among others, into what is now called 'prognostic groups', with the hope to personalize prognosis and treatment for a given patient and tumor. This is a big challenge, because there are many prognostic factors that we already know, but there are others that we still ignore.

\section{AME: In case of any divergence of opinion in the establishment of the staging system, how to deal with them?}

Prof. Rami-Porta: We can consider this at two different levels. In the IASLC SPFC, we have solved any discrepancies by consensus among its members. Changes introduced in the TNM classification must be clinically meaningful and not for the sake of taxonomy exclusively. Prognosis and differences in prognosis help in assigning 
descriptors to categories, and to group sets of tumors with similar prognosis into stages. But this is not all. For example, in the $8^{\text {th }}$ edition, clinical stage IIIC and clinical stage IVA have the same prognosis. So, they could be grouped together, but we chose not to do that because stage IIIC represents a locally advanced tumor and stage IVA defines metastatic disease within the chest. Both situations are anatomically different and need different approaches. So, it made sense to the SPFC members to keep them separately. Another example is M1a and M1b: they have the same prognosis, but, again, they represent different types of anatomic extent that have to be differentiated. Therefore, while they are grouped in the same stage IVA, they are assigned two different categories.

At clinical level, it is very easy to solve conflicting situations. General rule number 4 of the TNM classification says that when we are in doubt, we should assign the lower category or the lower stage to a given tumor.

\section{AME: What are the important factors in staging analysis? What could be the role of a surgeon in this regard?}

Prof. Rami-Porta: The most important factor is to have complete data. Over these past two decades, we have learnt that it is better to have a smaller database with very detailed data than a large one with incomplete information. Most data used to inform the $8^{\text {th }}$ edition were retrospective and incomplete in many aspects concerning the descriptors. However, there was a smaller database that had been collected prospectively and had the detailed information we needed to analyze certain descriptors. In fact, if we could say something new about the $M$ component of the classification, it was because the smaller, prospective database had the details on the number and location of the metastases. This allowed us to create a new category for multiple extrathoracic metastases (M1c) and to redefine M1b for those tumors with one extrathoracic metastasis, only. This is why by all means we will try to stimulate prospective registration of data for the $9^{\text {th }}$ edition.

The role of the surgeons in staging cannot be emphasized enough. As I mentioned above, the TNM classification is pure anatomy and anatomy is very important for surgeons. This is one of the reasons why we have an exceeding number of surgical patients compared to the number of patients treated by non-surgical means. The fact is that the surgical series that were submitted to the IASLC International Database were very rich in detail regarding anatomic descriptors. We lacked non-surgical cases. However, although the recommendations for changes in the $8^{\text {th }}$ edition of the TNM classification were based mainly on surgical data, a recent article by Chansky et al. published in the Fournal of Thoracic Oncology presented the external validation of the proposed changes based on a very large series of non-surgical patients from the National Cancer Database of the United States of America. So, the innovations of the $8^{\text {th }}$ edition apply to all tumors regardless of their therapeutic modality.

\section{Lung cancer staging and guidance in treatment}

AME: We noticed the IASLC 8th edition lung cancer staging system (only) included 790 Chinese cases of lung cancer. In China, there are numerous medical centers undertaking treatment for more than 1,000 cases of lung cancers per year. In your opinion, how can Chinese medical centers provide more qualified cases for subsequent lung cancer staging projects?

Prof. Rami-Porta: Yes, you are right. There are many institutions in China that treat thousands of patients every year. The doctors in those centers must be very busy. Participating in the IASLC Staging Project takes some time. The datasets are very complete and you need to fill the online forms as thoroughly as possible. In my opinion, the ideal is to have one responsible person to take care of the collection and registration of data. This person may be a doctor, but it can also be a well-trained data manager. Most data entered in the IASLC data forms are clinically relevant, so they should be easily available from admission and discharge records, and therapeutic (surgical or other) and pathology reports. But someone has to gather all these documents. If you have a digital clinical record system, it is much easier, but this system is not universally available.

Before embarking in the IASLC Staging Project, potential contributors must be convinced that it is important to do so: important for them, for the larger international medical community and, eventually, for the patients. After all, we all work for the benefit of our patients. Those who have already contributed or are contributing to the IASLC Staging Project have found it both personally and professionally rewarding. At a personal level, contributors constitute a very active community whose members interact among them at annual meetings and create personal links that will last all their lives. At the professional level, contributors participate in the furtherance of medical knowledge and feel they are integrated in a sound and solid scientific community that, so far, has produced very relevant results. 
AME: Do you have any suggestions in the construction of a lung cancer database? Which is the best representative to build the lung cancer database: Centers for Disease Control and Prevention, Medicare or medical centers?

Prof. Rami-Porta: Databases must have clear objectives. So, the first step is to think about what we want from the database. Registration of lots of data with no clear purpose is a waste of energy, time and resources. If one institution or service wants to create a database, it is important to know why they want it: will it be a merely administrative database or a clinical database? Will the objective be to study the TNM classification? If it is so, then it will be necessary to include all T, $\mathrm{N}$ and $\mathrm{M}$ descriptors. Will the objective be to study treatment complications? Then, all possible complications have to be listed and predefined for prospective collection of data. In summary, we must know what we want the database for. Once we know this, then, we must select the most appropriate data to try to answer a clinical or an administrative question. As I said before, it also is important to appoint one or two responsible persons to feed the database. Because doctors usually are very busy, the ideal is to have a properly trained data manager to do the job.

The particular case of the IASLC International Database has the advantage that contributors to the database can retrieve their own cases at any time. If they do not have a database, they can use the IASLC International Database as if it were their own. However, the IASLC International Database is focused on staging and other prognostic factors, and may not be adequate to study other aspects of lung cancer management.

It is difficult to say which of the existing databases is the best representative, because it depends on the objectives of the database. I can tell you for sure that the best representative for staging purposes is the IASLC International Database, because it was specifically designed to study staging. In general, administrative databases are not good enough to explore clinical questions. Specific clinical and research questions need specific databases.

AME: How to establish a unified, standardized operating procedure on lymph node dissection of lung cancer, especially in the 12-14 lymph nodes?

Prof. Rami-Porta: This is a difficult issue. An old adage says that 'two who say they do the same thing do not do the same thing'. Systematic nodal dissection, defined in 1996 during an international and multidisciplinary workshop on lung cancer staging, organized by Prof. Goldstraw under the auspices of the IASLC, that took place at the Royal Brompton Hospital in London, UK, is nowadays well described in books and in videos, especially the mediastinal part of it which is the surgeon's responsibility. However, nodal stations 12 to 14 can be both the domain of the thoracic surgeon, when performing segmentectomies or wedge resections, or of the pathologist, who will examine the lobectomy or pneumonectomy specimens. The only way to unify both the surgical and the pathologic procedures is to describe them well and then follow the descriptions scrupulously. Video-recording the operations and the pathologic examination may help find flaws in the procedures and, once these are identified, find solutions for a better standardization.

\section{AME: In the definition of M staging, M1a (pleural or pericardial nodules, thoracic or pericardial effusion, and contralateral or bilateral lobe nodules) did not change, but the oligometastatic lesions were independently classified, such as thoracic single organ single metastases were subdivided into M1b, whereas single or multiple organ multiple metastases were subdivided into M1c. Does this new staging method help us to make more aggressive treatment strategy-such as surgery-for this part of the oligometastatic patients?}

Prof. Rami-Porta: Well, when you name something, you make it more relevant. In the case of the $M$ component of the TNM classification, isolating single extrathoracic metastasis in a different category (M1b) will make them more evident. This can have, at least, two important consequences. Firstly, it may lead to redefine oligometastatic disease. Up to now, there is no single definition of this metastatic state: from 1 to 5 metastasis; some think they should be in the same organ; others, that they can be in different organs. So, there is no unified definition. And, secondly, these single extrathoracic metastases will probably be treated more intensively. We now have more means than a few decades ago to treat these lesions radically, that is, with intention to eliminate all known disease and cure it: radiotherapy, stereotactic radiotherapy, radiofrequency ablation, microwaves, high intensity focused ultrasounds, chemotherapy and targeted therapies, alone or in combination. Single brain and adrenal metastases have been surgically removed for decades and their 5 -year survival rates are quite reasonable (15\% and $25 \%$, respectively), considering that the usual 5 -year survival for stage IV is 
close to $0 \%$. However, we must be humble and recognize that the last word on the $M$ component has not been said, yet. We still need to know more. Just a few months after the publication in the Fournal of Thoracic Oncology of the original article on the $M$ component, a single-institution experience was published in the Fournal of Thoracic Disease. In that article, Dias et al. from Portugal showed that, while they could validate the $M$ categories of the $8^{\text {th }}$ edition TNM with their data, they found that the prognosis of one or two extrathoracic metastases was similar. So, more research and more data are needed to better understand metastatic disease.

\section{AME: AFCC, UICC and IASLC recommend at least six} lymph nodes resection during surgery to facilitate precise staging, including at least three N1 regional lymph nodes and three $\mathrm{N} 2$ regional lymph nodes. At the same time, if NO only based on $<6$ of the lymph node sampling, Staging and Prognostic Factors Committee (SPFC) members have different sounds: some members thought that can be identified as pNO, but some members believe that should be classified as pNO (un) to show its uncertainty. What is your opinion on this problem?

Prof. Rami-Porta: I am on the side of those who think that, when there are less than 6 lymph nodes removed and they are negative, the pN0 category should be qualified by the adjective 'uncertain'. We now know that quantifying nodal disease has prognostic relevance. The SPFC observed this both in the analyses of the $7^{\text {th }}$ edition and of the $8^{\text {th }}$ edition. For the $7^{\text {th }}$ edition, quantification was done by the number of involved nodal zones. For the $8^{\text {th }}$, it was done by the number of involved nodal stations. The more involved nodal zones and the more involved nodal stations, the worse the prognosis. Others have found the same counting the number of involved lymph nodes or calculating the lymph node ratio, that is, the number of involved lymph nodes divided by the number of removed lymph nodes. Others have also found that, even in pN0 tumors, the number of removed lymph nodes has prognostic impact. What all these findings are telling us is that the intraoperative lymph node evaluation must be as thorough as possible. I participated in the activities leading to the definition of lobe-specific systematic nodal dissection, but I do not believe in it. This type of dissection is a sort of sampling based on the probable routes of lymphatic dissemination depending on the lobar location of the primary tumor. It is pure statistics and statistics do not work for the individual patient. However, it is better than not exploring the lymph nodes at all, and it was precisely proposed to stimulate those who did not perform any type of intraoperative nodal assessment to start doing it with the hope that they would progress towards standard systematic nodal dissection. We also know, that even sub-centimeter tumors may have involved mediastinal lymph nodes. My recommendation is to perform systematic nodal dissection of the ipsilateral mediastinum, hilum and intrapulmonary lymph nodes in every case of lung cancer, admitting that, at the present state of knowledge, perhaps adenocarcinomas in situ and minimally invasive adenocarcinomas can be spared such a procedure. Systematic nodal dissection ensures that no regional lymph nodes are left behind and facilitates the identification of unexpected nodal disease for which adjuvant therapy has proved to be of significant benefit.

\section{Personal experience and inspiration in lung cancer research}

\section{AME: What is your source of inspiration in your study of lung cancer?}

Prof. Rami-Porta: There have been many sources. I went into medical school with the purpose to become a surgeon, but it was Prof. Juan Sánchez-Lloret Tortosa, from the University of Barcelona, the thoracic surgeon who taught us the thoracic lessons, who inspired me to choose thoracic surgery. I clearly remember his passionate presentations, always illustrated with real cases he had managed, sometimes in dramatic circumstances. Then, as a senior medical student, I was lucky enough to be accepted for clinical clerkships at the Department of Cardio-thoracic Surgery of the Medical University of South Carolina Hospital, in the USA, where I met Prof. Edward Parker. I saw the first thoracic procedures in my life there and I got really hooked to the specialty. Prof. Tsuguo Naruke also had an important influence on me. He was very active in staging issues that he could soundly support with the large experience of the National Cancer Center Hospital, where I had the chance to train for some time when I was a very young thoracic surgeon. During my residency years, I became very interested in lung cancer and, by extension, in thoracic oncology in general. Managing thoracic neoplasms is more than a mere technical procedure. It requires a lot of thinking, planning, and multidisciplinary decision-making discussions. It is, after all, a technical and intellectual challenge that I was ready to accept for the whole of my 
professional life. Finally, in these past two decades, Prof. Goldstraw has been a constant source of inspiration and encouragement to continue working in staging thoracic malignancies.

AME: If a medical student would like to study lung cancer, which field (surgery, radiology, pathology etc.) would you recommend for him/her to pursue? Why?

Prof. Rami-Porta: Confucius is credited for saying 'Choose a job you love and you will never have to work a day in your life'. Please, note that Confucius said 'a job you love', not 'a job with which you are in love'. Being in love is a serious pathologic state that frequently leads to disaster: we are magnetized by the profession or the person we are in love with and we do not see the whole reality behind. To love means to accept that profession or that person as they are, with their virtues and defects. We have to know well to love a profession or a person. So, the students who are interested in lung cancer must reflect on their own capabilities, and their objectives and expectations in live. Professional and personal lives must be integrated and there are specialties that make this quite difficult. Thoracic surgery is one of them. In any surgical specialty, you know when you start an operation, but you can never be sure when you will finish it. Many times, meetings and dinners have to be cancelled. Students must know that they will have to devote a lot of time if they want to master their specialty. Non-surgical specialties are less demanding and can be better scheduled in regular hours. As students or recent graduates, we do not know all the aspects of the specialty. It is important to visit departments, to talk to several specialists, to talk to residents to know their own views. At the end, the decision will be personal and very often based on incomplete information. In any case, whatever specialty they choose, if they study it with passion, I have no doubts that they will enjoy it, even if they were not so sure when they chose it. I am fortunate I can say that I have never regretted to have chosen thoracic surgery. It is a hard specialty, but I feel I am in permanent vacation, because I really love it, with its lights and its shadows.

All the specialties you mentioned-surgery, radiology, pathology, etc.-are critical to manage lung cancer patients. All are important in their own measure, and none is more important than the others. So, the students have to reflect on their own affinities with the particular pros and cons of each specialty and choose the one they love best.
AME: What do you think is the most important factor for a surgeon to become successful both in the clinical practice and the academic arena?

Prof. Rami-Porta: Success, as so many other things, is multifactorial. To become a successful surgeon, one has to know its profession well. This means to study hard, to practice regularly and to constantly update one's knowledge. The surgeon, as a specialist, is supposed to know a lot about his particular field, but must not forget to see the patient as a unique person with individual values and needs. The surgeon must do so with honesty, modesty and compassion. I have heard saying that modesty is a nice virtue, but that one goes much farther without it. It may be true in the short-run, but not in the long. Honesty and modesty pay off. Those who lack them may be temporary successful, but patients and colleagues will soon identify them and reject them. One must be honest to himself and to the patients. One must be honest in studies and publications. In our specialty, modesty is essential. We should never overemphasize our successes, because next day disaster may come. And when it comes, we have to accept it as part of our job and we must continue to work for the benefit of our patients. Feeling compassion is, in my opinion, part of our profession. Our patients are not only sick from the scientific point of view, but have a personal perception of their illness that also affects them individually and socially. We have to do our best to put ourselves in their shoes. I know that I may sound oldfashioned, but I still think that in the $21^{\text {st }}$ century, such old values as honesty, modesty and compassion will still prevail.

\section{AME: Thank you very much for sharing your insights!}

\section{Acknowledgements}

None.

\section{Footnote}

Conflicts of Interest: The authors have no conflicts of interest to declare.

(Editors: Grace S. Li, Jianfei Shen, JTD, jtd@amepc.org)

Cite this article as: Li GS, Shen J. The lung cancer research career of Prof. Ramón Rami-Porta: passion, vision and reflection. J Thorac Dis 2017;9(11):E1025-E1031. doi: 10.21037/jtd.2017.09.137 